\title{
VII. Zur analytisch-geometrischen Behandlung der Krystallographie.
}

\author{
Von
}

Th. Liebisch in Berlin.

(Fortselzung v. d. Zeitschr. I, 132.)

9.

Zwillingskrystalle.

Zu den merkwürdigsten Erscheinungen, welche die Krystalle darbieten, gehören diejenigen slabilen Gleichgewichtszustände krystallisirler Materie, welche unter dem Namen der Zwillingskrystalle bekannt sind. Wenn man die Flächen des einen Individuums eines $Z$ willings auf das Coordinatensystem des anderen Individuums bezieht, so bewahrt das Grundgesetz, welches die einfachen Krystalle beherrscht - das Gesetz der rationalen Indices - seine Gültigkeit nur noch in gewissen Gruppen der Zwillingskrystalle. Zwar hat Fr. Na umann den Satz aufgestellt, dass min a llen Zwillingskrystallen or th ö̈d ris cher Formen jede Fläche des einen Individuums, wenn sie auf das andere Individuum transponirt wird, einer krystallographischen Fläche dieses Individuums entspricht, indem sich ihro Parameter auch in dem Axensysteme dieses Individuums als rationale Mul-

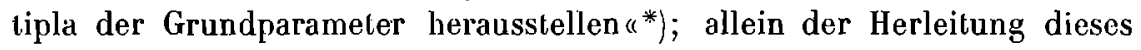
Satzes liegt die Hypothese von Ghr. S. We iss zu Grunde, derzufolge die Grundparameter (d. s. die Axeneinheiten) entweder durch rationale Zahlen oder durch Quadratwurzeln aus rationalen Zahlen darstellbar sind. Diese Hypothese befindet sich im Widerspruch mit den genauesten Winkelmessungen und mit den thermischen Eigenschaften der Krystalle. In Folge hiervon erleidet der $\mathrm{Naumann}$ 'sche Salz Einschränkungen: welche in dem Nachstehenden aufgesucht werden sollen.

*) Elemente der theorelischen Krystallographie. 1856. S. 67. 
Da bei der überwiegenden Mehrzahl der in der Natur vorkommenden Zwillingskrystalle die Zwillingsebene eine mögliche Krystallflache ist, so ist die Forderung aufgestellt worden, dass dic auf der Zwillingsaxe senkrceht stehende Fläche stets krystallographisch möglich sein müsse*). Die Untersuchungen über die Zwillingsbildungen der Krystalle des asymmetrischen Krystallisationssystemes, darunter insbesondere die umfassenden Forschungen G. vom Rath's über die Zwillinge der Plagioklase***), haben in uberzeugender Weise gelehrt, dass jene Forderung nicht festgehalten werden kann.

Nach Fr. Naumann ist "die Zwillingsaxe allgemein eine krystallographisch reelle Linie, also entweder eine der Axen der Krystallreihe, oder eine Kante, oder eine Flächennormale irgend einer ihrer Gestalten ( $\left.{ }^{* * *}\right)$. Es liegt der Bezeichnung: »krystallographisch reelle Linie « die Ansicht zu Grunde, dass eine mögliche Kante zugleich als Normale auf eine mögliche Fläche, und umgekehrt die Normale einer möglichen Fläche zugleich als eine mögliche Kante aufgefasst werden könne. Dass dies jedoch nicht immer der Fall ist, auch nicht, wie Na uman meinte "zum wenigsten in allen orthobasischen Krystallsystemen « soll zunächst nachgewiesen werden.

Aus den Untersuchungen über die Zwillinge asymmetrischer Krystalle geht hervor, dass die Angabe der Drehungsaxe in die Fassung des allgemeinen Gesetzes der Zwillingsbildung aufzunehmen ist. Demnach würde das Gesetz in Rede, auf alle beobachteten Fälle erweitert, so lauten :

"Zwei Individuen einer Krystallgattung bilden einen Zwilling, wenn sie derart verbunden sind, dass sie durch Drehung des einen Individuums um $180^{\circ}$ um eine gewisse, hinsichtlich ihrer Lagezu möglichen Flächen und Kanten der Krystallgattung bestimmbare Linie in parallele Stellung gebracht werden können $\alpha$.

Die nähere Bestimmung der Zwillingsebene und der Zwillingsaxe betreffend, so sind theoretisch vier Möglichkeiten, deren Vorkommen in der Natur durch die vorliegenden Beobachtungen $\dagger$ ) bestätigt wird, vorhanden:

1) Zwillingsebene und $Z$ willingsaxe besitzen rationale Indices;

2) die Zwillingsebene besitzt rationale Indices, die $Z$ willingsaxe nicht;

*) Vgl. V. v o n Lang, Lehrbuch der Krystallographie. 1866. S. 116. A. Sch ra uf, Physikal. Min. Bd. I. 1866. S. 8.

**) Pogg. Ann. Bd. 138. S. 449. Bd. 144. S. 219. Bd. 147. S. 22. 274. Erg.

Bd. VI. S. 378. - Monatsber. Berlin. Akad. 1876. 24. Febr.

***) Fr. Na u ma n ; Lehrbuch der reinen und angewandten Krystallographie. 1830.

Bcl. II. 202. - Der Satz ist wiederholt in: A. Schrauf, Physikal. Min. Bd. I. 1866.

S. 185. E. S. Dana, Text Book of Mineralogy. 1877. p. 89.

H) Vgl. A. S a de beck, Angewandte Krystallographie. 1876. 
3) die Zwillingsaxe besilzt rationale Indices, die Zwillingsebene nicht;

4.) weder die Zwillingsebene noch die Zwillingsaxe besitzt rationale Indices.

Es soll nun untersucht werden, wann der Fall 1, auf welchen sich der angeführte $\mathrm{Na}$ u mann' sche Satz bezieht, eintritt.

Die Beziehung, welche zwischen dèn Indices einer Fläche $H\left\{h_{1} h_{2} h_{3}\right\}$ und denjenigen ihrer Normale $\mathrm{H}\left[\eta_{1} \eta_{2} \eta_{3}\right]$ besteht, ergiebt sich aus den Formeln (43) und (45) Bd. I. S. 145 d. Zeitschr., wenn hierin an Stelle der Coordinaten Indices eingefuhrt werden. Es ist dabei zu berücksichtigen, dass die Coordinaten einer Fläche und diejenigen ihrer Normale mit gleichen Proportionalitätsfactoren zu versehen sind. Aus (43) folgt:

Aus (45) ergiebt sich:

$$
\begin{aligned}
& \sqrt{\Delta} \frac{\eta_{1}}{a_{2} a_{3}}=\Delta_{11} \frac{h_{1}}{a_{1}}+\Delta_{12} \frac{h_{2}}{a_{2}}+\Delta_{13} \frac{h_{3}}{a_{3}} \\
& \sqrt{\Delta} \frac{\eta_{2}}{a_{3} a_{1}}=\Delta_{21} \frac{h_{1}}{a_{1}}+\Delta_{22} \frac{h_{2}}{a_{2}}+\Delta_{23} \frac{h_{3}}{a_{3}} \\
& \sqrt{\Delta} \frac{\eta_{3}}{a_{1} a_{2}}=\Delta_{31} \frac{h_{1}}{a_{1}}+\Delta_{32} \frac{h_{2}}{a_{2}}+\Delta_{33} \frac{h_{3}}{a_{3}}
\end{aligned}
$$

$$
\begin{aligned}
& \sqrt{\Delta} \frac{h_{1}}{a_{1}}=c_{11} \frac{\eta_{1}}{a_{2} a_{3}}+c_{12} \frac{\eta_{2}}{a_{3} a_{1}}+c_{13} \frac{\eta_{3}}{a_{1} a_{2}} \\
& \sqrt{\Delta} \frac{h_{2}}{a_{2}}=c_{21} \frac{\eta_{1}}{a_{2} a_{3}}+c_{22} \frac{\eta_{2}}{a_{3} a_{1}}+c_{23} \frac{\eta_{3}}{a_{1} a_{2}} \\
& \sqrt{\Delta} \frac{h_{3}}{a_{3}}=c_{31} \frac{\eta_{1}}{a_{2} a_{3}}+c_{32} \frac{\eta_{2}}{a_{3} a_{1}}+c_{33} \frac{\eta_{3}}{a_{1} a_{2}}
\end{aligned}
$$

Aus (89) und (90) gewinnt man folgende Relation zwischen den Indices einer Fläche und denen ihrer Normale :

$$
\sum_{i k=1}^{3} \Delta_{i k} \frac{h_{i}}{a_{i}} \frac{h_{k}}{a_{k}}=\sum_{i k \lambda \mu=1}^{3} \Delta_{i k} \frac{c_{i \lambda} a_{\lambda} \eta_{\lambda}}{a_{1} a_{2} a_{3} \sqrt{\bar{\Delta}}} \frac{c_{k \mu} a_{\mu} \eta_{\mu}}{a_{1} a_{2} a_{3} \sqrt{\bar{\Delta}}}
$$

oder, wenn man die quadratischen Formen

$$
\begin{gathered}
\sum_{i k=1}^{3} A_{i k} \frac{h_{i}}{a_{i}} \frac{h_{k}}{a_{k}} \\
\frac{1}{a_{1}^{2} a_{2}{ }^{2} a_{3}{ }^{2}} \sum_{i k=1}^{3} c_{i k} a_{i} a_{k} \eta_{i} \eta_{k}
\end{gathered}
$$

beziehungsweise mit $D(h h)$ und $K(\eta \eta)_{i}^{\text {bezeichnet: }}$

$$
D(h h)=K(\eta \eta) \text {. }
$$

Wie die Gleichungen (89) und (90) erkennen lassen, verhalten sich im a symmetrischen System weder die Indices der Normale einer möglichen Krystallfläche noch die Indices der Normalebene einer möglichen Krystallkante wie rationale Zahlen. Demnach kann bei den Zwillingen dieses Systems der Fall 1 nicht eintreten. 
Im monos ymmetrischen Krystallisationssysteme ist:

$$
\begin{aligned}
\left(x_{2} x_{3}\right) & =90^{\circ},\left(x_{3} x_{1}\right)=\beta,\left(x_{1} x_{2}\right)=90^{\circ} \\
c_{23} & =c_{12}=0, c_{31}=\cos \beta . \\
\Delta & =\left|\begin{array}{ccc}
1 & 0 & \cos \beta \\
0 & 1 & 0 \\
\cos \beta & 0 & 1
\end{array}\right|=1-\cos ^{2} \beta . \\
\Delta_{11} & =\Delta_{33}=1, \quad \Delta_{22}=1-\cos ^{2} \beta \\
\Delta_{23} & =\Delta_{12}=0, \quad \Delta_{31}=-\cos \beta .
\end{aligned}
$$

Demnach erhalten wir aus (89) :

und aus $(90)$ :

$$
\begin{aligned}
& \sin \beta \frac{\eta_{1}}{a_{2} a_{3}}=\frac{h_{1}}{a_{1}}-\cos \beta \frac{h_{3}}{a_{3}} \\
& \sin \beta \frac{\eta_{2}}{a_{3} a_{1}}=\sin ^{2} \beta \frac{h_{2}}{a_{2}} \\
& \sin \beta \frac{\eta_{3}}{a_{1} a_{2}}=-\cos \beta \frac{h_{1}}{a_{1}}+\frac{h_{3}}{a_{3}}
\end{aligned}
$$

$$
\begin{aligned}
& \sin \beta \frac{h_{1}}{a_{1}}=\frac{\eta_{1}}{a_{2} a_{3}}+\cos \beta \frac{\eta_{3}}{a_{1} a_{2}} \\
& \sin \beta \frac{h_{2}}{a_{2}}=-\frac{\eta_{2}}{a_{3} a_{1}} \\
& \sin \beta \frac{h_{3}}{a_{3}}=\cos \beta \frac{\eta_{1}}{a_{2} a_{3}}+\frac{\eta_{3}}{a_{1} a_{2}}
\end{aligned}
$$

Aus den Formeln (92) und (93) erhellt, dass im monosymmetrischen Systeme nur die Indices der Normale der Fläche $\{010\}$ und die Indices der Normalebene der Kante [010] sich wie rationale Zahlen verhalten. Da $\{010\}$ als Symmetrieebene nicht Zwillingsebene sein kann, so kann der Fall 1 auch bei den Zwillingsbildungen monosymmetrischer Krystalle nicht vorkommen.

Im rhombischen System ist :

$$
\begin{gathered}
\left(x_{2} x_{3}\right)=\left(x_{3} x_{1}\right)=\left(x_{1} x_{2}\right)=90^{\circ} \\
c_{23}=c_{31}=c_{12}=0, \Delta=1 \\
\Delta_{11}=\Delta_{22}=\Delta_{33}=1, \Delta_{23}=\Delta_{31}=\Delta_{12}=0
\end{gathered}
$$

Mit Rücksicht hierauf gehen die Relationen (89) und (90) über in:

$$
\begin{aligned}
\frac{\eta_{1}}{a_{2} a_{3}} & =\frac{h_{1}}{a_{1}} \\
\frac{\eta_{2}}{a_{3} a_{1}} & =\frac{h_{2}}{a_{2}} \\
\frac{\eta_{3}}{a_{1} a_{2}} & =\frac{h_{3}}{a_{3}}
\end{aligned}
$$


Die Verhältnisse der Indices sind:

$$
\begin{aligned}
& \eta_{1}: \eta_{2}: \eta_{3}=\frac{h_{1}}{a_{1} a_{1}}: \frac{h_{2}}{a_{2} a_{2}}: \frac{h_{3}}{a_{3} a_{3}} \\
& h_{1}: h_{2}: h_{3}=a_{1}^{2} \eta_{1}: a_{2}^{2} \eta_{2}: a_{3}^{2} \eta_{3}
\end{aligned}
$$

Hieraus ergiebt sich, dass nur die Indices der Normalen der Flächen $\{100\},\{010\},\{001\}$ und die Normalebenen der Kanten [100], [010], [001] sich wie rationale Zahlen verhalten. Daher ist der Fall 1 auch bei den holoëdrischen Krystallen des rhombischen Systems nicht möglich. Er kann nur bei den Zwillingsbildungen hemiëdrischer und hemimorpher Krystalle eintreten.

Im tetragonale n Systeme ist:

$$
a_{1}=a_{2}=a, a_{3}=1
$$

Demnach geht (94) uber in :

$$
\begin{aligned}
\frac{\eta_{1}}{a} & =\frac{h_{1}}{a} \\
\frac{\eta_{2}}{a} & =\frac{h_{2}}{a} \\
\frac{\eta_{3}}{a a} & =h_{3}
\end{aligned}
$$

Die Verhältnisse der Indices sind :

$$
\begin{aligned}
& \eta_{1}: \eta_{2}: \eta_{3}=h_{1}: h_{2}: a^{2} h_{3} \\
& h_{1}: h_{2}: h_{3}=\eta_{1}: \eta_{2}: \frac{\eta_{3}}{a a}
\end{aligned}
$$

Hieraus geht hervor, dass im tetragonalen System die Indices der Normalen der Flächen $\left\{h_{1} h_{2} 0\right\}$ aus der Verticalzone, die Indices der Normale der Fläche $\{001\}$, die Indices der zu den horizontalen Kantenrichtungen $\left[\eta_{1} \eta_{2} 0\right]$ normal stehenden Flächen und die Indices der Normalebene der Kante [001] sich wie rationale Zahlen verhalten. Demgemäss gehören zu dem Falle 1 die Zwillingsbildungen holoëdrischer Krystalle, bei denen eine Fläche eines ditetragonalen Prismas Zwillingsebene ist, und ausserdem diejenigen Zwillingsbildungen hemiëdrischer *), tetartoëdrischer und hemimorpher Krystalle, bei denen eine Fläche eines ditetragonalen Prismas oder eine Symmetrieebene des Systems Zwillingsebene ist.

Legen wir der Betrachtung des hexagonalen Systems orthobexagonale Axen zu Grunde, so ist in (94):

zu setzen, so dass:

$$
a_{1}=a, a_{2}=a \sqrt{3}, a_{3}=1
$$

$$
\frac{\eta_{1}}{a \sqrt{3}}=\frac{h_{1}}{a}
$$

*) Vgl. P. Grolh, Physikalische Krystallographie. 1876. $§ 107$. 


$$
\begin{aligned}
\frac{\eta_{2}}{a} & =\frac{h_{2}}{a \sqrt{3}} \\
\frac{\eta_{3}}{a a \sqrt{3}} & =h_{3}
\end{aligned}
$$

Die Verhältnisse der Indices sind:

$$
\begin{aligned}
& \eta_{1}: \eta_{2}: \eta_{3}=3 h_{1}: h_{2}: 3 a^{2} h_{3} \\
& h_{1}: h_{2}: h_{3}=\eta_{1}: 3 \eta_{2}: \frac{\eta_{3}}{a a}
\end{aligned}
$$

Hieraus ist ersichtlich, dass für die Formen des hexagonalen Systems ein dem so eben für das tetragonale System abgeleiteten Satze analoger Satz besteht. Es verhalten sich auch im hexagonalen Systeme die Indices der Normalen der Flächen aus der Verticalzone, die Indices der Normale der Basis, die Indices der zu den horizontalen Kanten normal stehenden Flächen und die Indices der Normalebene der verticalen Kantenrichtung wie rationale Zahlen. Daher gehören zu dèm Falle 1 die Zwillinge holoëdrischer Krystalle, bei denen eine Fläche eines dihexagonalen Prismas Zwillingsebene ist, ferner die Zwillinge hemiëdrischer ${ }^{*}$ ), tetartoëdrischer und hemimorpher Krystalle, bei denen eine Fläche eines dihexagonalen Prismas oder eine Symmetrieebene des Systems Zwillingsebene ist.

Im regulär en System ist :

Demnach folgt aus (94):

$$
a_{1}=a_{2}=a_{3}=1
$$

$$
\eta_{1}=h_{1}, \eta_{2}=h_{2}, \eta_{3}=h_{3}
$$

Im regulären System, und nur in diesem, sind die Indices der Normale einer möglichen Krystallfläche stets gleich den Indices dieser Fläche, und umgekehrt die Indices der Normalebene einer möglichen Kante stets gleich den Indices dieser Kante. Die Zwillinge dieses Systems gehören daher sämmtlich dem Falle 1 an.

Die analytisch-geometrische Darstellung der Theorie der Zwillingskrystalle ist von Naumann, Sénarmont, Sella und Schrauf bearbeitet worden.

Fr. Naumann hat diese Theorie »auf die symmetrische Copulation zweier identischer Axensysteme gegrundet und als ein allgemeines Problem der analytischen Geometrie behandelt (**). Es sei gestatlet seine Resultate anzufuhren und eine Vergleichung seiner Bezeichnungsweise mit der hier angewendeten beizufugen.

*) Vgl. P. Groth, a. a. 0. $\S 106$, 344. 377.

**) Elemente der theoretischen Krystallographie. 1856. S. 62. 135. 179. 256.298. 
Naumann.

1) Die von den Axen $x, y, z$ eingeschlossenen Winkel:

$$
(y z)=\alpha,(z x)=\beta,(x y)=\gamma .
$$

2) Quadrat des Sinus der Ecke (xyz):

$$
\begin{gathered}
E=1-\cos ^{2} \alpha-\cos ^{2} \beta-\cos ^{2} \gamma+ \\
2 \cos \alpha \cos \beta \cos \gamma .
\end{gathered}
$$

3) Die von den Axenebenen eingeschlossenen, beziehungsweise an den Axen $x, y, z$ liegenden Winkel :

$$
\begin{gathered}
A, B, C . \\
\text { 4) } \sin ^{2} \alpha, \sin ^{2} \beta, \sin ^{2} \gamma \\
A^{\prime}=\cos \alpha-\cos \beta \cos \gamma=\cos A \sin \beta \sin \gamma \\
B^{\prime}=\cos \beta-\cos \gamma \cos \alpha=\cos B \sin \gamma \sin \alpha \\
C^{\prime}=\cos \gamma-\cos \alpha \cos \beta=\cos C \sin \alpha \sin \beta \\
\text { 5) Parameter (Axenabschnitte) der Fläche } F \text { : }
\end{gathered}
$$

$$
a: b: c
$$

Sind die Grundparameter (Axenlängen :)

$$
\mathbf{a}: \mathbf{b}: \mathbf{c}
$$

so ist :

$$
a: b: c=m \mathbf{a}: n \mathbf{b}: r \mathbf{c} .
$$

Die Ableitungszablen

$$
m: n: r
$$

sind umgekehrt proportional den Indices.

6) Die Parameter (axoparallelen Abstände von den Axenebenen, Coordinaten) eines Punktes einer durch den Mittelpunkt des Axensystems gehenden Geraden :

$$
\mu: \nu: \varrho
$$

7) Verhältnisse der Parameter $p: q: s$ der Centronormale einer Fläche $\boldsymbol{F}(a: b: c)$ :

$$
\begin{gathered}
p: q: s= \\
b c \sin ^{2} \alpha-a b B^{\prime}-c a C^{\prime}: \\
c a \sin ^{2} \beta-b c C^{\prime}-a b A^{\prime}: \\
a b \sin ^{2} \gamma-c a A^{\prime}-b c B^{\prime}
\end{gathered}
$$

$$
\begin{gathered}
\left(x_{2} x_{3}\right),\left(x_{3} x_{1}\right),\left(x_{1} x_{2}\right) \\
\cos \left(x_{i} x_{k}\right)=c_{i k} \\
\Delta=\left|\begin{array}{lll}
c_{11} & c_{12} & c_{13} \\
c_{21} & c_{22} & c_{23} \\
c_{31} & c_{32} & c_{33}
\end{array}\right| \\
\\
\left(x_{1}\right),\left(x_{2}\right),\left(x_{9}\right) \\
\Delta_{11}, \Delta_{22}, \Delta_{33} \\
\Delta_{23} \\
\Delta_{31} \\
\Delta_{12}
\end{gathered}
$$

$$
\begin{gathered}
\frac{1}{u_{1}}: \frac{1}{u_{2}}: \frac{1}{u_{3}}=\frac{a_{1}}{h_{1}}: \frac{a_{2}}{h_{2}}: \frac{a_{3}}{h_{3}} \\
a_{1}: a_{2}: a_{3}
\end{gathered}
$$

$$
\frac{1}{h_{1}}: \frac{1}{h_{2}}: \frac{1}{h_{3}}
$$

$$
\begin{gathered}
\xi_{1}: \xi_{2}: \xi_{3}= \\
\frac{\eta_{1}}{a_{2} a_{3}}: \frac{\eta_{2}}{a_{3} a_{1}}: \frac{\eta_{3}}{a_{1} a_{2}}
\end{gathered}
$$

Formel (43)

Bd. I. S. 145 . 
Naumann.

8) Parameter einer Fläche $F(a: b: c)$ ausgedrückt durch die Parameter $p: q: s$ ihrer Centronormale :

$$
\begin{aligned}
& b c E=p+q \cos \gamma+s \cos \beta \\
& c a E=q+s \cos \alpha+p \cos \gamma \\
& a b E=s+p \cos \beta+q \cos \alpha
\end{aligned}
$$

Formel (45)

Bd. I. S. 145 .

Naumann berechnete zunächst die Transformationsgleichungen mit Hülfe deren die Axen des zweiten Individuums auf das Axensystem des ersten Individuums bezogen werden (a. a. 0. S. $69 \mathrm{f}$., S. $377 \mathrm{f}$ ), wenn die Parameter der Zwillingsebene $(a: b: c)$ und damit auch diejenigen der Zwillingsaxe $(p: q: s)$ gegeben sind. Die Parameter der Axen $x^{\prime}, y^{\prime}, z^{\prime}$ des zweiten Individuums sind in dem Schema enthalten:

worin :

$$
\begin{array}{rlll} 
& x & y & z \\
x^{\prime}: \mu & q & s \\
y^{\prime}: p & \nu & s \\
z^{\prime}: p & q & \varrho
\end{array}
$$

$$
\begin{aligned}
& \mu=\frac{p^{2}-q^{2}-s^{2}-2 q s \cos \alpha}{2 b c E} \\
& \nu=\frac{q^{2}-s^{2}-p^{2}-2 s p \cos \beta}{2 c a E} \\
& \varrho=\frac{s^{2}-p^{2}-q^{2}-2 p q \cos \gamma}{2 a b E}
\end{aligned}
$$

Die Transformationsgleichungen, durch welche die Parameter einer beliebigen Fläche $F^{\prime}\left(a^{\prime}: b^{\prime}: c^{\prime}\right)$ des zweiten Individuums auf das Axensystem des ersten Individuums bezogen werden; hat $\mathrm{Na}$ uman nur für orthoëdrische Axensysteme abgeleitet. Die transformirten Parameter $a_{1}: b_{1}: c_{1}$ der Fläche $F^{\prime}$ sind (S. 67 f.):

worin :

$$
\begin{aligned}
& a_{1}=\frac{a^{\prime} b^{\prime} c^{\prime} S}{2 a b c\left(b b^{\prime}+c c^{\prime}\right) b^{\prime}-A b^{\prime} c^{\prime}} \\
& b_{1}=\frac{a^{\prime} b^{\prime} c^{\prime} S}{2 a b c\left(c c^{\prime}+a a^{\prime}\right) b^{\prime}-B c^{\prime} a^{\prime}} \\
& c_{1}=\frac{a^{\prime} b^{\prime} c^{\prime} S}{2 a b c\left(a a^{\prime}+b b^{\prime}\right) c^{\prime}-C a^{\prime} b^{\prime}}
\end{aligned}
$$

$$
\begin{aligned}
& S=a^{2} b^{2}+c^{2} a^{2}+b^{2} c^{2} \\
& A=-a^{2} b^{2}-c^{2} a^{2}+b^{2} c^{2} \\
& B=-a^{2} b^{2}+c^{2} a^{2}-b^{2} c^{2} \\
& C=a^{2} b^{2}-c^{2} a^{2}-b^{2} c^{2}
\end{aligned}
$$

Naumann gelangte zu diesem Resultat, indem er die Coordinaten der Durchschnittspunkte einer Fläche $F^{\prime}$ des zweiten Individuums mit den Axen 
dieses Individuums bezogen auf das Axensystem des ersten Individuums berechnete und hierauf die Parameter der Fläche, welche durch die erwähnten drei Punkte geht, aufsuchte. Die so gewonnenen Grössen sind die Parameter der auf das erste Axensystem transportirten Fläche $F^{\prime}$. Diesen indirecten Weg musste $\mathrm{N}$ a u man $\mathrm{n}$ einschlagen, weil er die allgemeine Transformation der Coordinaten nicht durchgeführt hatte.

Eine vollständigere Behandlung der Aufgabe, welche sich Nau man $\mathrm{n}$ gestellt hatte, verdanken wir $Q u$. Sella*). Derselbe bezeichnet die Axeneinheiten mit $a, b, c$ und setzt :

$$
a \cos (a b)=a^{\prime}, b \cos (b c)=b^{\prime}, c \cos (c a)=c^{\prime} .
$$

Die Fläche, deren Axenabschnitte sich wie die Längen $O M: O N$ : $O P$ verhalten, hat das Symbol $m n p$ :

$$
m: n: p=\frac{a}{O M}: \frac{b}{O N}: \frac{c}{O P} \text {. }
$$

Die Kante, welche Diagonale eines Parallelepipedons mit den Kantenverhältnissen $O H: H K: K L$ ist, hat das Symbol $[h k l]$ :

$$
h: k: l=\frac{O H}{a}: \frac{H K}{b}: \frac{K L}{c} .
$$

Die Indices der Ebene $m^{\prime} n^{\prime} p^{\prime}$, welche senkrecht zur Kante [mnp] steht, verhalten sich wie:

$$
\begin{gathered}
m^{\prime}: n^{\prime}: p^{\prime}=m a^{2}+n a^{\prime} b+p c^{\prime} a: n b^{2}+p b^{\prime} c+m a^{\prime} b \\
: p c^{2}+m c^{\prime} a: n b^{\prime} c .
\end{gathered}
$$

Die Indices der Geraden [mnp], welche normal zur Fläche $m^{\prime} n^{\prime} p^{\prime}$ steht, verhalten sich wie:

$$
\begin{gathered}
m: n: p= \\
\left|\begin{array}{lll}
m^{\prime} & a^{\prime} b & c^{\prime} a \\
n^{\prime} & b b & b^{\prime} c \\
p^{\prime} & b^{\prime} c & c c
\end{array}\right|:\left|\begin{array}{lll}
a a & m^{\prime} & c^{\prime} a \\
a^{\prime} b & n^{\prime} & b^{\prime} c \\
c^{\prime} a & p^{\prime} & c c
\end{array}\right|:\left|\begin{array}{lll}
a a & a^{\prime} b & m^{\prime} \\
a^{\prime} b & b b & n^{\prime} \\
c^{\prime} a & b^{\prime} c & p^{\prime}
\end{array}\right|
\end{gathered}
$$

Bei Sella bedeutet $m^{\prime} n^{\prime} p^{\prime}$ die Zwillingsebene, $[m n p]$ die Zwillingsaxe. Die Bezeichnungen von Naumann und Sella können in folgender Weise gegenubber gestellt werden:

Naumann.

1) Axeneinheiten:

$$
\mathbf{a}: \mathbf{b}: \mathbf{c}
$$

2) Parameter der Zwillingsfläche

$$
a: b: c
$$

3) Parameter der Zwillingsaxe:

$$
p: q: s \text {. }
$$

$$
\begin{gathered}
\text { Sella. } \\
a: b: c \\
\frac{a}{m^{\prime}}: \frac{b}{n^{\prime}}: \frac{c}{p^{\prime}} \\
a m: b n: c p .
\end{gathered}
$$

*) Sul boro adamantino. Mem. della R. Accademia delle sc. di Torino. Serie II. tom XVII. Nota (B). 1857. 
Sella leitete Nota (B) p. 42 die Verhiltnisse der Indices ab, welche Jen Axen des zweiten Individuums in Bezug auf das Axensystem des ersten Individuums zukommen:

$$
\begin{gathered}
\frac{m^{2} a^{2}-n^{2} b^{2}-p^{2} c^{2}-2 n p b^{\prime} c}{2\left(m a^{2}+n a^{\prime} b+p c^{\prime} a\right)}: n: p \\
m: \frac{n^{2} b^{2}-p^{2} c^{2}-m^{2} a^{2}-2 p m c^{\prime} a}{2\left(n b^{2}+p b^{\prime} c+m a^{\prime} b\right.}: p \\
m: n: \frac{p^{2} c^{2}-m^{2} a^{2}-n^{2} b^{2}-2 m n a^{\prime} b}{2\left(p c^{2}+m c^{\prime} a+n b^{\prime} c\right)} .
\end{gathered}
$$

In dieses mit $(\mathfrak{2})$ ubereinstimmende Resultat fuhrte $\mathrm{S}$ ella die Indices der Zwillingsebene $m^{\prime} n^{\prime} p^{\prime}$ ein, indem er die Indices $m^{\prime}, n^{\prime}, p^{\prime}$ e inzeln g le ic h den in (ङ) angegebenen Werthen setzte. Nun sind diese Werthe jenen Indices nur bis auf einen Proportionalitätsfactor gleich. Daher sind von hier ab die Formeln Sella's nicht mehr richtig. Um die von S ella gestellte Aufgabe zu lösen muss man für die Grössen, welche in (ฐ) rechts stehen, ihre wirklichen Werthe, die aus Formel (90) zu entnehmen sind, eintragen.

Bei der Berechnung der Verhältnisse der Indices einer Fläche des einen Individuums bezogen auf das Axensystem des anderen Individuums benutzte $\mathrm{S}$ ell a die eleganten Formeln, welche er für die allgemeine Transformation der Coordinaten *) erhalten hatte. Das Resultat (Nota (B) p. 43) ist ebenfalls mit dem angedeuteten Fehler behaftet.

A. Schrauf**) unterwarf die analytisch-geometrische Theorie der Zwillingskrystalle einer Bearbeitung nach der Methode, welche von H. de Sénarmon $t^{* * *}$ ) angegeben, aber nur auf das reguläre System ausgedehnt worden ist.

Sénarmon $\mathrm{t}$ bezeichnet die Axen der beiden Individuen mit $X, Y, Z$ und $X^{\prime} Y^{\prime} Z^{\prime}$, den Pol der Zwillingsebene mit $P$, die Pole entsprechender Flächen mit $Q$ und $Q^{\prime}$. Dann ist :

$$
\begin{array}{r}
\cos X P Q=\frac{\cos Q X-\cos P X \cos P Q}{\sin X P \sin P Q}= \\
-\cos X P Q^{\prime}=-\frac{\cos Q^{\prime} X-\cos P X \cos P Q^{\prime}}{\sin X P \sin P Q^{\prime}}
\end{array}
$$

woraus folgt:

(F) $\cos Q^{\prime} X=2 \cos P X \cos P Q-\cos Q X$.

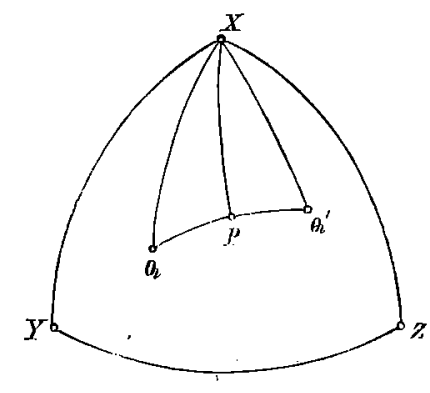

*) 1. c. Nota (A). Sul congiamento di assi in un sistema cristallino.

***) Sitzungsber. Wien. Akad. Bd. L. I. Abth. Math. naturw. Cl. 1864. Physikal. Min. Bd. I. 1866. S. 185.

***j Sur quelques groupements de cristaux du système régulier. Annales des mines.

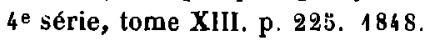


In analoger Weise erhält man :

$$
\begin{aligned}
& \cos Q^{\prime} Y=2 \cos P Y \cos P Q-\cos Q Y \\
& \cos Q^{\prime} Z=2 \cos P Z \cos P Q-\cos Q Z .
\end{aligned}
$$

Insbesondere ergiebt sich hieraus:

$$
\begin{aligned}
& \cos X^{\prime} Y=2 \cos P Y \cos P X-\cos X Y \\
& \cos X^{\prime} Z=2 \cos P Z \cos P X-\cos X Z \\
& \cos Y^{\prime} Z=2 \cos P Z \cos P Y-\cos Y Z \\
& \cos X^{\prime} X=2 \cos ^{2} P X-1 \\
& \cos Y^{\prime} Y=2 \cos ^{2} P Y-1 \\
& \cos Z^{\prime} Z=2 \cos ^{2} P Z-1 .
\end{aligned}
$$

Man erhält aus (F) die Lösungen der Transformationsaufgabe, mit der sich Naumann und Sella beschäftigt haben, wenn man die Werthe der Cosinus aus (35) entnimmt.

Die Symbole der in Betracht kommenden Flächen seien :

Dann ist :

$$
P\left\{z_{1} z_{2} z_{3}\right\}, \quad Q\left\{h_{1} h_{2} h_{3}\right\}, \quad Q^{\prime}\left\{h_{1}^{\prime} h_{2}^{\prime} h_{3}^{\prime}\right\} \text {. }
$$

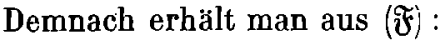

$$
\begin{aligned}
& \cos P Q=\frac{D(z h)}{\sqrt{D(z z) D(h h)}} \\
& \cos P X=\frac{\sqrt{\Delta} \cdot \frac{z_{1}}{a_{1}}}{\sqrt{D(z z)}} \\
& \cos Q X=\frac{\sqrt{\Delta} \cdot \frac{h_{1}}{a_{1}}}{\sqrt{D(h h)}} .
\end{aligned}
$$

$$
\frac{D(z z) \sqrt{D}(h h)}{\sqrt{\Delta}}-\cos Q^{\prime} X=2 D(z h) \frac{z^{\prime}}{a_{r}}-D(z z) \frac{h^{\prime}}{a_{r}}
$$

oder :

$$
\rho h_{1}^{\prime}=2 D(z h) \frac{z_{1}}{a_{1}}-D(z z) \frac{h_{1}}{a_{1}}
$$

worin $\varrho$ einen Proportionalitätsfactor bedeutet. Analoge Ausdrucke ergeben sich für $\varrho h_{2}^{\prime}$ und $\varrho h^{\prime}{ }_{3}$.

A. Schrauf hat die von Séna rmont angedeuteten Operationen in umgekehrter Reihenfolge ausgeführt. Er berechnet zuerst die Cosinus der von den Coordinatenaxen $X, Y, Z$ und $X^{\prime}, Y^{\prime}, Z^{\prime}$ eingeschlossenen Winkel(Formel 103) S. 189), hierauf die Indices $x_{1} x_{2} x_{3}, y_{1} y_{2} y_{3}, z_{1} z_{2} z_{3}$ der zu den Axen $X^{\prime}, Y^{\prime}, Z^{\prime}$ des zweiten Individuums normal stehenden Flächen bezogen auf das Axensystem $X, Y, Z$ des ersten Individuums (Formel (110) S. 191), dann die Cosinus der Winkel, welche eine beliebige Fläche $M\{u v w\}$ des ersten Individuums mit den Flächen $\left\{x_{1} x_{2} x_{3}\right\},\left\{y_{1} y_{2} y_{3}\right\}$, $\left\{z_{1} z_{2} z_{3}\right\}$ einschliesst (Formel (111) S. 192). Hieraus folgen schliesslich die 
Indices $u^{\prime} v^{\prime} w$ der Fläche $M$ bezogen auf das Axensystem $X^{\prime}, Y^{\prime}, Z^{\prime}$ des zweiten Individuums (Formel (113) S. 193).

Zur Vergleichung sei bemerkt, dass :

$$
A_{1}=\Delta, M_{1}=a_{1}^{2} a_{2}^{2} a_{2}^{2} D(z z) \text {. }
$$

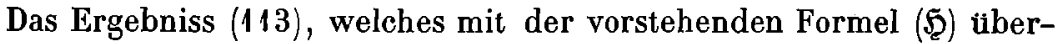
einstimmen musste, ist nicht richtig. Es soll $\cos M X^{\prime}$ in dem schiefwinkligen Axensysteme $X, Y, Z$ dargestellt werden durch die Winkel, welche $M$ und $X^{\prime}$ mit den Axen $X, Y, Z$ einschliessen. Dazu dient die Formel (17) S. 110. Es ist aus den angefuhrten Publicationen nicht ersichtlich, weshalb Schrauf an Stelle dieser Formel die folgende unrichtige Formel gewählt hat. Da nämlich :

$$
\begin{aligned}
& u=a \cos M X, v=b \cos M Y, w=c \cos M Z \\
& u^{\prime}=a \cos M X^{\prime}, v^{\prime}=b \cos M Y^{\prime}, w^{\prime}=c \cos M Z^{\prime}
\end{aligned}
$$

so lautet $(113)$ :

$$
a \cos M X^{\prime}=\frac{a \cos M X \cos X^{\prime} X+b \cos M Y \cos X^{\prime} Y+c \cos M Z \cos X^{\prime} Z}{\sqrt{\left[\cos ^{2} X^{\prime} X+\cos ^{2} X Y+\cos ^{2} X^{\prime} Z\right]}}
$$

So sind in der vorliegenden Literatur die Aufgaben, welche sich die analytisch-geometrische Theorie der Zwillingskrystalle zu stellen hat, nicht vollständig oder nicht einwurfsfrei behandelt worden. Es möchte daher nicht uberflüssig erscheinen, wenn im Folgenden nochmals eine analytische Darstellung dieser Theorie versucht wird.

Vorausgesetzt wird, dass die Elemente der Krystallgattung - die Win-. kel $\left(x_{2} x_{3}\right),\left(x_{3} x_{1}\right),\left(x_{1}^{1} x_{2}\right)$ und die Verhältnisse $a_{1}: a_{2}: a_{3}-$ und die Indices der Zwillingsfläche $Z\left\{z_{1} z_{2} z_{3}\right\}$ gegeben seien. Die Beziehungen zwischen den Indices $z_{1}, z_{2}, z_{3}$ und den Indices der Zwillingsaxe $Z\left[\zeta_{1} \zeta_{2} \zeta_{3}\right]$ sind :

$$
\begin{aligned}
\sqrt{\boldsymbol{\Delta}} \frac{\zeta_{k} a_{k}}{a_{1} a_{2} a_{3}} & =\sum_{i=1}^{3} \Delta_{i k} \frac{z_{i}}{a_{i}} \\
\sqrt{\boldsymbol{\Delta}} a_{1} a_{2} a_{3} \frac{z_{k}}{a_{k}} & =\sum_{i=1}^{3} c_{i k} a_{i} \zeta_{i}
\end{aligned}
$$

Ferner ist nach $(91)$ :

$$
D(z z)=K(\zeta \zeta)
$$

I. Wenn die Indices einer Fläche $H\left\{h_{1} h_{2} h_{3}\right\}$ des ersten Individuums bekannt sind, so sollen die Indices der jener Fläche $H$ correspondirenden Fläche $H^{\prime}\left\{h_{1}^{\prime} h_{2}^{\prime} h_{3}^{\prime}\right\}$ des zweiten Individuums bezogen auf das Coordinatensystem des ersten Individuums berechnet werden.

Da die Zwillingsfläche $Z$ den von den Flächen $H$ und $H^{\prime}$ eingeschlossenen Winkel $\left(H H^{\prime}\right)$ halbirt, so stehen die Goordinaten der drei Flächen $Z, H, H^{\prime}$, welche wir beziehungsweise mit $p_{1} p_{2} p_{3}, u_{1} u_{2} u_{3}, u_{1}^{\prime} u_{2}^{\prime} u_{3}^{\prime}$ 
Th. Liebisch.

bezeichnen nach S. $149 \mathrm{Bd}$. I. d. Zeitschr. in folgender Beziehung zu einander :

oder :

$$
\varrho p_{i}=u_{i}+u_{i}
$$

worin :

$$
-u_{i}^{\prime}=u_{i}-\varrho p_{i}
$$

$$
\varrho=\frac{\sin \left(H^{\prime} H\right)}{\sin \left(H^{\prime} Z\right)}
$$

Die Winkel $\left(H^{\prime} H\right)$ und $\left(H^{\prime} Z\right)$ können durch den Winkel $(H Z)$, der unter den angegebenen Voraussetzungen als bekannt anzusehen ist, ausgedrückt werden. Es ist :

$$
\begin{gathered}
\left(H^{\prime} Z\right)=(Z H) \\
\left(H^{\prime} H\right)=\left(H^{\prime} Z\right)+(Z H)=2(Z H)
\end{gathered}
$$

Demnach erhalten wir :

$$
\varrho=\frac{\sin 2(Z H)}{\sin (Z H)}=2 \cos (Z H)
$$

oder nach(35) mil Rücksicht auf die oben $\mathrm{S} .76$ eingeführten Bezeichnungen :

so dass :

$$
\varrho=\frac{2 D(z h)}{\sqrt{D(z z) D(h h)}}
$$

$$
u_{i}^{\prime}=\frac{2 D(z h)}{\sqrt{D(z z) D(h h)}} p_{i}-u_{i}
$$

Führen wir an die Stelle der Coordinaten $p_{i}, u_{i}, u_{i}^{\prime}$ die Indices $z_{i}, h_{i}$, $h^{\prime}{ }_{i}$ ein, so ist zu setzen :

$$
p_{i}=\varrho \frac{z_{i}}{a_{i}}, \quad u_{i}=\varrho^{\prime}, \frac{h_{i}}{a_{i}}, \quad u_{i}^{\prime}=\varrho^{\prime \prime} \frac{h_{i}^{\prime}}{a_{i}}
$$

und die Werthe der Proportionalitătsfactoren sind aus (34) zu entnehmen:

$$
\varrho=\frac{V \bar{\Delta}}{V D(z z)}, \quad \varrho^{\prime}=\frac{\sqrt{\Delta}}{V D(h h)}
$$

So ergeben sich die gesuchten Transformationsgleichungen :

$$
\begin{gathered}
\sigma h_{1}^{\prime}=2 D(z h) z_{1}-D(z z) h_{1} \\
\sigma h^{\prime}=2 D(z h) z_{2}-D(z z) h_{2} \\
\sigma h_{3}^{\prime}=2 D(z h) z_{3}-D(z z) h_{3}
\end{gathered}
$$

worin $\sigma$ einen Proportionalitätsfactor bedeulet.

Zwischen einander entsprechenden Flächen $I$ und $H^{\prime}$ besteht eine umkehrbare Beziehung, d. h. sucht man zu $I I$ die correspondirende Fläche, so findet man $H^{\prime}$ aus (101), und sucht man zu $H^{\prime}$ die correspondirende Fläche, so kommt man durch nochmalige Anwendung von (101) wieder auf $H$ zurück. In der That, setzt man in :

$$
\sigma h_{i}=\dot{2} D\left(z h^{\prime}\right)_{z i}-D(z z) h_{i}^{\prime}
$$

für die Indices $h_{i}^{\prime}$ ihre Werthe nach (101) ein, so erhält man :

$$
\sigma h_{i}=2 z_{i}\{2 D(z z) D(z h)-D(z z) D(z h)\}-D(z z)\left\{2 D(z h) z_{i}-D(z z) h_{i}\right\}
$$


oder :

(102)

$$
\sigma h_{i}=D^{2}(z z) h_{i}
$$

d. h. man erhält wieder $h_{i}$, wenn man von dem constanten Factor $D^{2}(z z)$, der aus den Verhältnissen $h_{1}: h_{2}: h_{3}$ herausfällt, absieht.

Aus den Gleichungen (101) ergiebt sich, dass diejenigen Flächen, welche der Zone der Zwillingsaxe angehören, beiden Individuen des Zwillings gemein sind. Denn die Indices einer so beschaffenen Fläche $\left\{x_{1} x_{2} x_{3}\right\}$ erfüllen die Bedingungsgleichung:

welche nach (98) ubergeht in :

$$
x_{1} \zeta_{1}+x_{2} \zeta_{2}+x_{3} \zeta_{3}=0
$$

$$
\sum_{i k=1}^{3} \Delta_{i k} \frac{z_{i}}{a_{i}} \frac{x_{k}}{a_{k}}=D(z x)=0 .
$$

Mit Rucksicht hierauf folgt aus (101):

$$
\sigma x_{i}^{\prime}=-D(z z) x_{i}
$$

d. h. die Indices derjenigen Fläche, welche der Fläche $\left\{x_{1} x_{2} x_{3}\right\}$ entspricht, sind den Indices $x_{1}, x_{2}, x_{3}$ proportional, oder m. a. W. die beiden Flächen fallen in eine zusammen.

II. Wenn die Indices einer Kante $\mathrm{H}\left[\eta_{1} \eta_{2} \eta_{3}\right]$ des ersten Individuums bekannt sind, so sollen die Indices der jener Kante entsprecheñden Kante $H^{\prime}\left[\eta_{1}^{\prime} \eta_{2}^{\prime} \eta_{3}^{\prime}\right]$ des zweiten Individuums bezogen auf das Coordinatensystem des ersten Individuums berechnet werden.

Die Zwillingsaxe $\mathrm{Z}$ halbirt den Winkel $\left(\mathrm{HH}^{\prime}\right)$. Wir können also die Lösung der vorliegenden Aufgabe mit Hülfe der Zwillingsaxe in analoger Weise durchfuhren wie die Lösung der vorhergehenden Aufgabe unter Benutzung der Zwillingsfläche. Wir erhalten:

$$
\begin{aligned}
& \tau \eta_{1}^{\prime}=2 K(\zeta \eta) \zeta_{1}-K(\zeta \zeta) \eta_{1} \\
& \tau \eta_{2}^{\prime}=2 K(\zeta \eta) \zeta_{2}-K(\zeta \zeta) \eta_{2} \\
& \tau \eta_{3}^{\prime}=2 K(\zeta \eta) \zeta_{3}-K(\zeta \zeta) \eta_{3}
\end{aligned}
$$

worin $x$ einen Proportionalitätsfactor bedeutet.

Auch zwischen einander entsprechenden Kanten $\mathrm{H}$ und $\mathrm{H}^{\prime}$ besteht eine $\mathrm{umkehrbar}$ e Beziehung, wie daraus erhellt, dass :

mit Hïlfe von $(104)$ in :

$$
\tau \eta_{i}=2 K\left(\zeta \eta^{\prime}\right) \zeta_{i}-K(\zeta \zeta) \eta_{i}
$$

ubergefüh rt .wird.

$$
\tau \eta_{i}=K^{2}(\zeta \zeta) \eta_{i}
$$

Die der Zwillingsebene parallel laufenden Kanten des einen Individuums haben zu entsprechenden Kanten des anderen Individuums solche, welche den ersteren Kanten parallel gehen. Denn eine mil der Fläche $Z$ parallele Kante $\left[\xi_{1} \xi_{2} \xi_{3}\right]$ befriedigt die Gleichung :

oder nach $(99)$ :

$$
z_{1} \xi_{1}+z_{2} \xi_{2}+z_{3} \xi_{3}=0
$$




$$
\sum_{i k=1}^{3} c_{i k} a_{i} a_{k} \zeta_{i} \xi_{k}=0
$$

so dass :

$$
K(\zeta \xi)=0
$$

Daher ergiebt sich aus (104):

$$
\tau \boldsymbol{\xi}_{i}^{\prime}=-K(\zeta \zeta) \xi_{i}
$$

d. h. die Indices derjenigen Kante, welche der Kante $\left[\xi_{1} \xi_{2} \xi_{3}\right]$ entspricht, sind den Indices $\xi_{1}, \xi_{2}, \xi_{3}$ proportional.

III. Es soll der Winkel berechnet werden, den eine Fläche $H\left\{h_{1} h_{2} h_{3}\right\}$ des einen Individuums mit einer Fläche $L\left\{l_{1} l_{2} l_{3}\right\}$ des anderen Individuums eines Zwillings einschliesst.

Die Indices der Fläche $L$ bezogen auf das Axensystem des ersten Individuums seien $l_{1}^{\prime}, l_{2}^{\prime}, l_{3}^{\prime}$, dann ist nach $(35)$ :

$$
\cos \left(H L^{\prime}\right)=\frac{D\left(h l^{\prime}\right)}{\sqrt{D(h h) D\left(l^{\prime} l^{\prime}\right)}}
$$

oder, wenn man für die Indices $l_{1}^{\prime}, l_{2}^{\prime}, l_{3}^{\prime}$ ihse Werthe nach (101) einträgt:

$$
\cos \left(H L^{\prime}\right)=\frac{2 D(z h) D(z l)-D(z z) D(h l)}{D(z z) \sqrt{D(h h) D(l l)}}
$$

Insbesondere ergiebt sich hieraus der Cosinus des Winkels, welchen die einander entsprechenden Flächen $H$ und $H^{\prime}$ einschliessen :

$$
\cos \left(H H^{\prime}\right)=\frac{2 D^{2}(z h)-D(z z) D(h h)}{D(z z) D(h h)}
$$

IV. Es soll der Winkel berechnet werden, den eine Kante $\mathrm{H}\left[\boldsymbol{\eta}_{1} \eta_{2} \eta_{3}\right]$ des einen Individuums mit einer Kante $\Lambda\left[\lambda_{1} \lambda_{2} \lambda_{3}\right]$ des anderen Individuums eines $\mathrm{Zwillings} \mathrm{einschliesst.}$

Die Indices der Kante $\Lambda$ bezogen auf das Axensystem des ersten Individuums seien $\lambda_{1}^{\prime}, \lambda_{2}^{\prime}, \lambda_{3}^{\prime}$, dann ist nach $(40)$ :

$$
\cos \left(\mathrm{H} \Lambda^{\prime}\right)=\frac{K\left(\eta \lambda^{\prime}\right)}{\sqrt{K}(\eta \eta) K\left(\lambda^{\prime} \lambda^{\prime}\right)}
$$

oder, wenn man für die Indices $\lambda_{1}^{\prime}, \lambda_{2}^{\prime}, \lambda_{3}^{\prime}$ ihre Werthe nach (104) einträgt:

$$
\cos \left(\mathrm{H} \boldsymbol{A}^{\prime}\right)=\frac{\operatorname{a} \boldsymbol{K}(\zeta \eta) K(\zeta \lambda)-K(\zeta \zeta) K(\eta \lambda)}{K(\zeta \zeta) V^{\prime K}(\eta \eta) K(\lambda \lambda)}
$$

Insbesondere ergiebt sich hieraus der Cosinus des Winkels, welchen die einander entsprechenden Kanten $H$ und $H^{\prime}$ einschliessen :

$$
\cos \left(\mathrm{H}^{\prime}\right)=\frac{2 K^{2}(\zeta \eta)-K(\zeta \zeta) K(\eta \eta)}{K(\zeta \zeta) K(\eta \eta)}
$$

Aus den Formeln (101) bis (110) fliessen durch Specialisirung folgende Ergebnisse.

Aus (101) erhalten wir die Verhältnisse der Indices der Axenebenen $X_{1}^{\prime}, X_{2}^{\prime}, X_{3}^{\prime}$ des zweiten Individuums bezogen auf das Axensystem des 
ersten Individuums, indem wir für $h_{1} h_{2} h_{3}$ successive 100, 010, 001 eintragen. Der Kürze wegen möge noch:

$$
\frac{z_{\mu}}{a_{\nu}} \sum_{i=1}^{3} \Delta_{i \nu} \frac{z_{i}}{a_{i}}=g_{\mu \nu}
$$

gesetzt werden. Die gesuchten Indices verhalten sich wie:

$$
\begin{array}{llll}
\left.X^{\prime}{ }^{\prime}\right) & 2 g_{11}-D(z z): & 2 g_{21} & : 2 g_{31} \\
\left.X^{\prime}{ }_{2}\right) & 2 g_{12} & : 2 g_{22}-D(z z) & : 2 g_{32} \\
\left.X^{\prime}{ }_{3}\right) & 2 g_{13} & : 2 g_{23} & : 2 g_{33}-D(z z)
\end{array}
$$

Aus (104) erhalten wir die Verhältnisse der Indices der Axen $x^{\prime}{ }_{1}, x^{\prime}{ }_{2}$, $x^{\prime}{ }_{3}$ des zweiten Individuums bezogen auf das Axensystem des ersten Individuums, indem wir fur $\eta_{1} \eta_{2} \eta_{3}$ successive $100,010,001$ eintragen. Wir bedienen uns der abgekürzten Bezeichnung :

$$
\frac{\zeta_{\mu} a_{\nu}}{a_{1}^{2} a_{2}^{2} a_{3}^{2}} \sum_{i=1}^{3} c_{i \nu} a_{i} \zeta_{i}=\gamma_{\mu \nu}
$$

Dann verhalten sich die gesuchten Indices wie:

$$
\begin{aligned}
& \left.x_{1}^{\prime}\right) \quad 2 \gamma_{11}-K(\zeta \zeta): 2 \gamma_{21} \quad: 2 \gamma_{31} \\
& \left.x_{2}^{\prime}\right) \quad 2 \gamma_{12} \quad: 2 \gamma_{22}-K(\zeta \zeta): 2 \gamma_{32} \\
& \left.x^{\prime}{ }_{3}\right) \quad 2 \gamma_{13} \quad: 2 \gamma_{23} \quad: 2 \gamma_{33}-K(\zeta \zeta)
\end{aligned}
$$

Aus (108) ergeben sich Ausdrucke für die Cosinus der Winkel, welche die Axenebenen $X_{1}, X_{2}, X_{3}$ des einen Individuums mit den Axenebenen $X_{1}^{\prime}, X_{2}^{\prime}, X_{3}^{\prime}$ des anderen Individuums einschliessen:

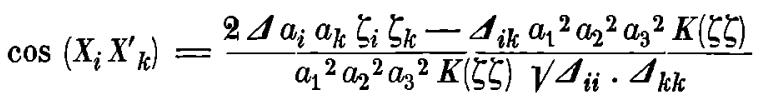

Aus (110) folgen Ausdrücke für die Cosinus der Winkel, welche die Axen $x_{1}, x_{2}, x_{3}$ des einen Individuums mit den Axen $x_{1}^{\prime}, x_{2}^{\prime}, x_{3}^{\prime}$ des anderen Individuums einschliessen:

$$
\cos \left(x_{i} x^{\prime}{ }_{k}\right)=\frac{2 \Delta \frac{z_{i} z_{k}}{a_{i} a_{k}}-\cos \left(x_{i} x_{k}\right) D(z z)}{D(z z)},
$$

Die Transformationsgleichungen (101) und (104) gelten für das a s y mmet ris ch e System; aus ihnen sollen jetzt die entsprechenden Formeln fưr die übrigen Krystallisationssysteme abgeleitet werden.

Im monosymmetrischen System ist:

$$
\begin{aligned}
\sigma h_{i}^{\prime}= & 2\left\{\frac{z_{1} h_{1}}{a_{1} a_{1}}+\sin ^{2} \beta \frac{z_{2} h_{2}}{a_{2} a_{2}}+\frac{z_{3} h_{3}}{a_{3} a_{3}}-\cos \beta \frac{z_{1} h_{3}+z_{3} h_{1}}{a_{1} a_{3}}\right\} z_{i} \\
& -\left\{\frac{z_{1} z_{1}}{a_{1} a_{1}}+\sin ^{2} \beta \frac{z_{2} z_{2}}{a_{2} a_{2}}+\frac{z_{3} z_{3}}{a_{3} a_{3}}-2 \cos \beta \frac{z_{1} z_{3}}{a_{1} a_{3}}\right\} h_{i}
\end{aligned}
$$

und

$$
\begin{aligned}
\tau \eta_{i}^{\prime} & =2\left\{a_{1}^{2} \zeta_{1} \eta_{1}+a_{2}^{2} \zeta_{2} \eta_{2}+a_{3}^{2} \zeta_{3} \eta_{3}+\cos \beta a_{1} a_{3}\left(\zeta_{1} \eta_{3}+\zeta_{3} \eta_{1}\right)\right\} \zeta_{i} \\
& -2\left\{a_{1}^{2} \zeta_{1}^{2}+a_{2}^{2} \zeta_{2}^{2}+a_{3}^{2} \zeta_{3}^{2}+2 \cos \beta a_{1} a_{3} \zeta_{1} \zeta_{3}\right\} \eta_{i}
\end{aligned}
$$


Im rhombischen System ist :

$$
\begin{aligned}
\sigma h_{i}^{\prime} & =2\left\{\frac{z_{1} h_{1}}{a_{1} a_{1}}+\frac{z_{2} h_{2}}{a_{2} a_{2}}+\frac{z_{3} h_{3}}{a_{3} a_{3}}\right\} z_{i}-\left\{\frac{z_{1} z_{1}}{a_{1} a_{1}}+\frac{z_{2} z_{2}}{a_{2} a_{2}}+\frac{z_{3} z_{3}}{a_{3}} a_{3}\right\} h_{i} \\
\tau \eta_{i}^{\prime} & =2\left\{a_{1}^{2} \zeta_{1} \eta_{1}+a_{2}^{2} \zeta_{2} \eta_{2}+a_{3}{ }^{2} \zeta_{3} \eta_{3}\right\} \zeta_{i} \\
& -\left\{a_{1}{ }^{2} \zeta_{1}{ }^{2}+a_{2}{ }^{2} \zeta_{2}{ }^{2}+a_{3}{ }^{2} \zeta_{3}{ }^{2}\right\} \eta_{i}
\end{aligned}
$$

Im te tragonalen System ist :

$$
\sigma h_{i}^{\prime}=2\left\{z_{1} h_{1}+z_{2} h_{2}+a^{2} z_{3} h_{3}\right\} z_{i}-\left\{z_{1}^{2}+z_{2}^{2}+a^{2} z_{3}^{2}\right\} h_{i}
$$

(120) $\tau \eta_{i}^{\prime}=2\left\{a^{2} \zeta_{1} \eta_{1}+a^{2} \zeta_{2} \eta_{2}+\zeta_{3} \eta_{3}\right\} \zeta_{i}-\left\{a^{2} \zeta_{1}^{2}+a^{2} \zeta_{2}{ }^{2}+\zeta_{3}{ }^{2}\right\} \eta_{i}$.

Im hexagonalen System ist bei Zugrundelegung von orthohexagonalen Axen :

(121) $\sigma h_{i}^{\prime}=2\left\{3 z_{1} h_{1}+z_{2} h_{2}+3 a^{2} z_{3} h_{3}\right\} z_{i}-\left\{3 z_{1}^{2}+z_{2}^{2}+3 a^{2} z_{3}^{2}\right\} \mathrm{h}_{i}$

(122) $\tau \eta_{i}^{\prime}=\left\{a^{2} \zeta_{1} \eta_{1}+3 a^{2} \zeta_{2} \eta_{2}+\zeta_{3} \eta_{3}\right\} z_{i}-\left\{a^{2} \zeta_{1}{ }^{2}+3 a^{2} \zeta_{2}{ }^{2}+\zeta_{3}{ }^{2}\right\} \eta_{i}$.

Im regulären System ist:

$$
\begin{aligned}
& \sigma h^{\prime}=2\left\{z_{1} h_{1}+z_{2} h_{2}+z_{3} h_{3}\right\} z_{i}-\left\{z_{1}{ }^{2}+z_{2}{ }^{2}+z_{3}{ }^{2}\right\} h_{i} \\
& \tau \eta_{i}^{\prime}=2\left\{\zeta_{1} \eta_{1}+\zeta_{2} \eta_{2}+\zeta_{2} \eta_{3}\right\} \zeta_{i}-\left\{\zeta_{1}{ }^{2}+\zeta_{2}{ }^{2}+\zeta_{3}{ }^{2}\right\} \eta_{i} .
\end{aligned}
$$

Die Formeln (101), 104 und (115) bis (124) dienen zu Beantworlung der Frage nach den Fällen, in denen die auf das Axensystem des einen Individuums bezogenen Flächen der beiden Individuen eines Zwillings dem Gesetz der rationalen Indices unterworfen sind.

Es gehören hierher:

1) Die Zwillinge des regulären Systems*). .

2) Diejenigen $Z$ willinge des tetragonalen Systems, bei denen eine Fläche $\left\{z_{1} z_{2} 0\right\}$ aus der Verticalzone oder die Basis $\{001\}$ Zwillingsebene ist.

3) Diejenigen Zwillinge des hexagonalen Systems, bei denen eine Fläche $\left\{z_{1} z_{2} 0\right\}$ aus der Verticalzone oder die Basis $\{001\}$ Zwillingsebene ist.

4) Diejenigen $Z$ willinge des rhombischen Systems, bei denen $\{100\}$, $\{010\}$ oder $\{001\}$ Zwillingsebene ist.

Daraus sind zugleich die Einschränkungen ersichtlich, welche der im Eingange erwähnte Satz von Fr. N a uman n erleidet.

*) Vgl. Sénarmont a. a. 0. 